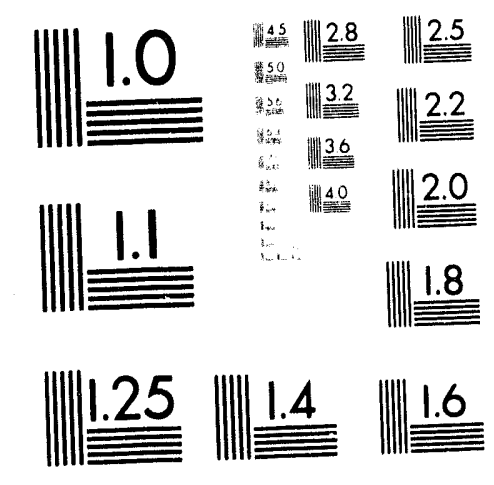



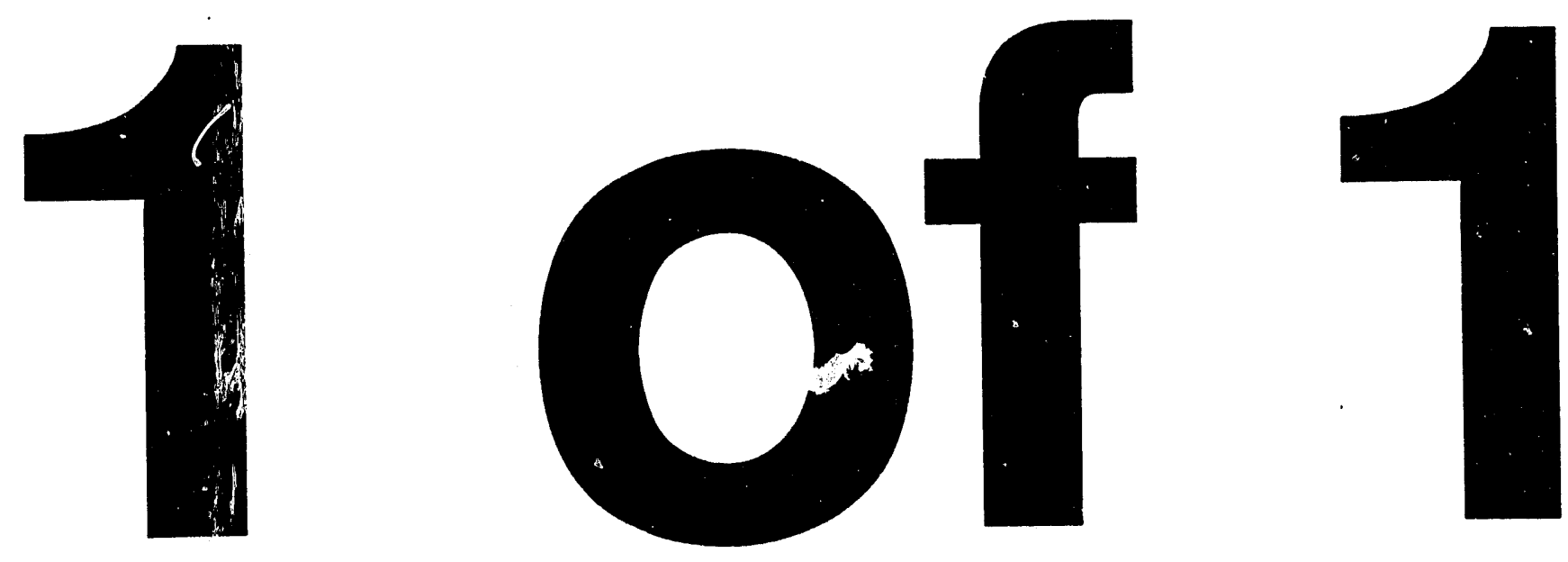
GA-A21398

\title{
MEASUREMENT OF ELECTRON \\ TEMPERATURE AND DENSITY \\ IN THE DIII-D NEUTRAL BEAM \\ ION SOURCE ARC CHAMBER
}

\author{
by \\ D.H. KELLMAN, J. BUSATH, and R. HONG
}

This is a preprint of a paper to be presented at the 15th IEEE Symposium on Fusion Engineering, October 11-15, 1993, Cape Cod, Massachusetts, and to be printed in the Proceedings.

\author{
Work supported by \\ U.S. Department of Energy \\ Contract DE-AC03-89ER51114
}

GENERAL ATOMICS PROJECT 3466 OCTOBER 1993

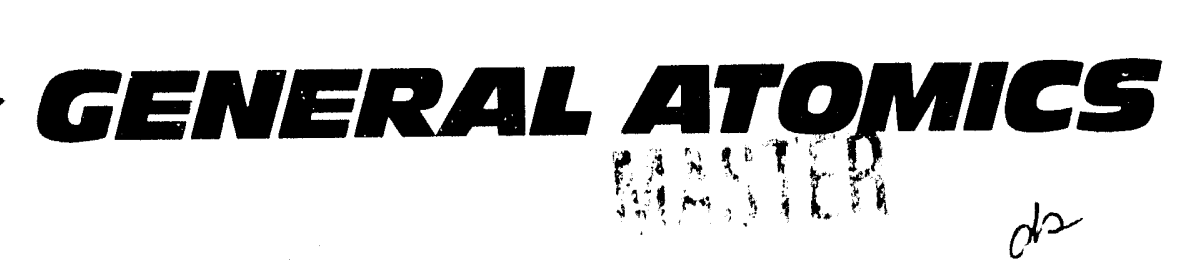

ULSTRIBUTION OF THIS DOCUMENT IS UNLIMITED 


\title{
MEASUREMENT OF ELECTRON TEMPERATURE AND DENSITY IN THE DIII-D NEUTRAL BEAM ION SOURCE ARC CHAMBER
}

\author{
D.H. Kellman, J. Busath, and R. Hong \\ General Atomics \\ P.O. Box 85608, San Diego, California 92186-9784
}

\begin{abstract}
A swept-bias Langmuir probe diagnostic was employed with the DIII--D neutral beam ion source in an effort to study the effects of filament temperature, arc power, and backstreaming energetic electrons on the electron temperature and density of the arc discharge inside the ion source arc chamber. The arc chamber contains six Langmuir probes biased with a negative dc voltage. These probes provide a feedback signal for regulation of the arc power supply, and give a relative indication of plasme uniformity within the arc chamber. For this study, one probe was reconnected to a voltage-sweeping power supply, and probe current versus voltage characteristics were generated. These characteristics provided the information necessary to calculate electron temperature and density. With arc discharge only, the results demonstrated that as filament temperature increases, so does electron density. Electron temperature decreases at a faster rate, however, as required to maintain constant ion saturation current (regulated by the arc power supply). The results also demonstrated that increasing arc power (through control of the arc power supply) results in higher electron temperature and density. Experiments were also performed with probe voltage sweeps during beam extraction, at various accelerator voltage levels and at different delay times after beam turn on with a fixed acceleration voltage. These results indicated an increase in electron temperature and density as acceleration voltage is increased. However, nearly identical trends result when arc discharges are produced at the same parameter settings as during these beams, but without beam extraction. This indicates minimal influence of backstreaming energetic electrons on electron temperature and density in the arc chamber. Temperature and density also remain fairly constant over time during a long beam pulse. These results help to provide a better understanding of ion source dynamics, and may lead to future improvements in operation.
\end{abstract}

\section{INTRODUCTION}

Since the adoption of the Common Long Pulse Source at DIII-D for use in neutral beam plasma heating, many improvements in performance of these ion sources have been implemented $[1,2]$. These improviments were brought about primarily through experimentation with various control schenies and operational modes, using waveforms from power supplies and fixed-voltage Langmuir probes as indications of the processes within the ion source.

To better understand the observations in terms of the physics involved, and to provide more insight leading to future improvements, a Langmuir probe on one of the ion sources has been utilized as a diagnostic tool, yielding data from which electron temperature and density within the ion source arc chamber is derived. This technique involves sweeping the Langmuir probe bias voltage from a negative value to a positive one while recording current measured in the probe. From the current versus voltage characteristics thus generated, electron temperature and density are calculated. These numbers can provide a better explanation of interactions between ion source controls and waveforms observed during operations.

\section{EXPERIMENTAL APPARATUS}

In early operation, the six Langmuir probes in the ion source arc chamber were biased with a negative dc voltage to measure ion saturation current. The probes provided an indication of an arc discharge, as well as a relative indication of spatial variation in plasma density within the arc discharge. The signal from one Langmuir probe was also utilized for feedback regulation of the arc power supply to improve ion source performance [2].

For this experiment, the bias circuitry of one Langmuir probe was replaced with equipment to provide the necessary voltage sweep, as well as signals representing probe voltage and current (Fig. 1). A trigger pulse transmitted to the equipment initiates a voltage ramp on the probe (typically, from -100 to $+100 \mathrm{~V}$ in $100 \mathrm{msec}$ ). Voltage and current data are transmitted to a digitizing oscilloscope where they are stored for future processing and analysis.

\section{INTERPRETATION OF DATA}

When voltage on the Langmuir probe is swept from -100 to $+200 \mathrm{~V}$, a classical Langmuir probe current/ voltage characteristic is generated as shown in Fig. 2. Of particular note (for the purpose of this experiment) are the ion saturation region, the ion saturation current intercept $I_{0}$, and the transition region.

Langmuir probe theory is well known, and has been described elsewhere [3]. According to this theory, electron temperature $T_{e}$ can be determined by subtracting ion saturation curreat from the Langmuir probe characteristic data, selecting two points $\left(I_{1}, V_{1}\right)$ and $\left(I_{2}, V_{2}\right)$ in the transition region of the resulting curve, and solving the equation

Marmecript received October 12, 1993. This is a report oi work sponsored by the U.S. Department of Energy under Contract No. DE-AC03-89ER51114. 


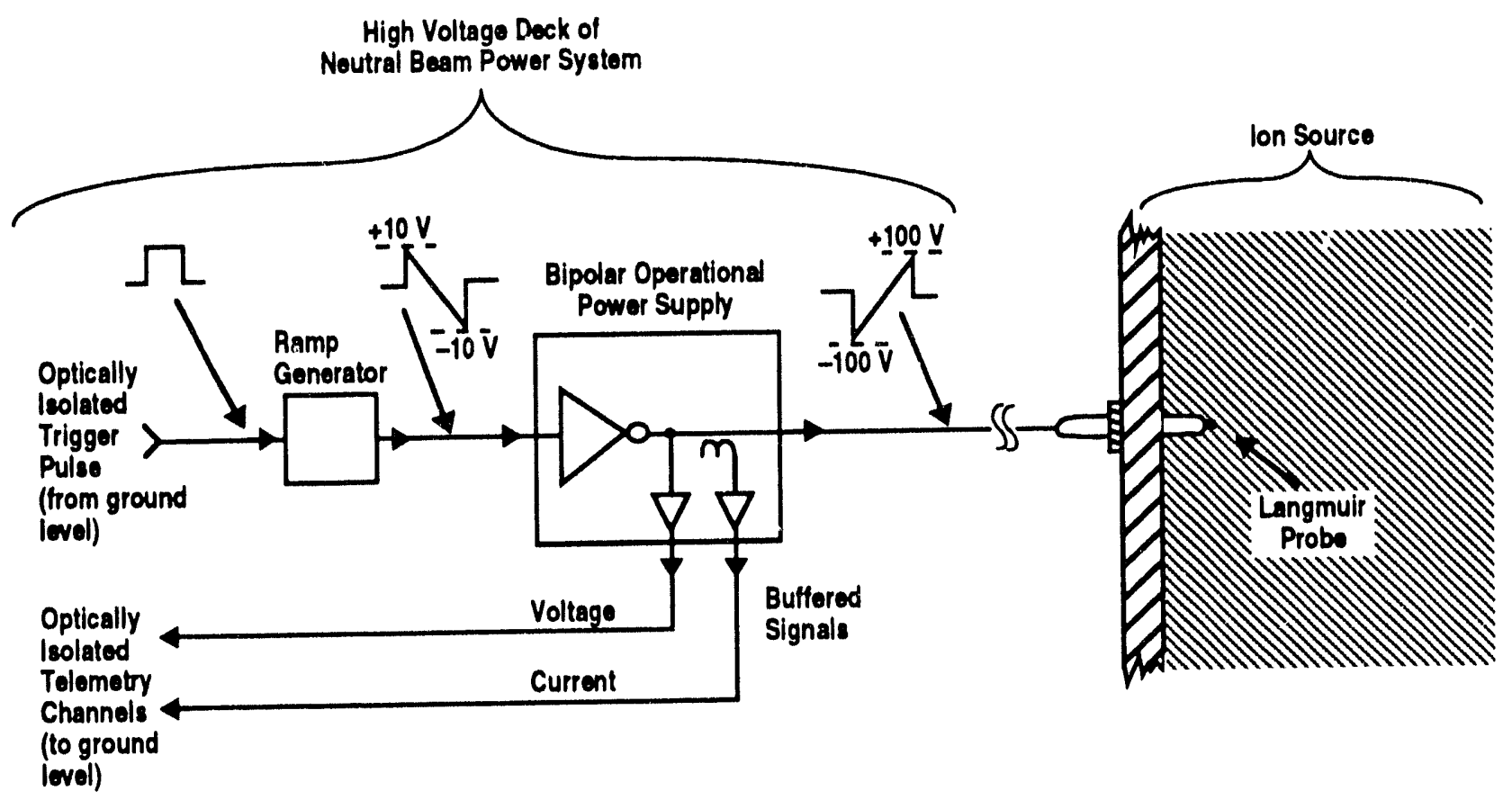

FIG. 1. Voltage-sweeping bias circuitry for Langmuir probe.

$$
T_{\mathrm{e}}=\frac{V_{2}-V_{1}}{\ln \left(I_{2} / I_{1}\right)}
$$

For a deuterium plasma and a probe surface area of $11 \mathrm{~mm}^{2}$, electron density $n$ is then given by

$$
n=2.066 \times 10^{17} \frac{I_{0}}{\sqrt{T_{e}}} .
$$

The scatter of the results calculated using (1) and (2) above is due to signal noise, power supply ripple, digitizing noise, and other experimental uncertainties.

\section{EFFECT OF FILAMENT TEMPERATURE}

To investigate the effect of filament temperature on electron temperature and density, a series of arc discharges was generated within the arc chamber with various values of voltage applied to the filaments. All other ion source operation parameters were maintained constant, and arc discharges at particular settings were repeated to check consistency of measurements made. When discharge equilibrium was achieved, the Langmuir probe voltage was swept from approximately -100 to $+100 \mathrm{~V}$, and probe current and voltage data were collected and stored. The data was later processed as described above.

The calculated values of temperature and density for each arc discharge are shown in Fig. 3, plotted against filament voltage. The graphs show that, as filament heating is increased, electron temperature in the plasma decreases, while density increases slightly. Within the range of data acquired, the relationships of temperature and density to filament voltage appear to be linear. The rate of increase in density appears much smaller than the rate of decrease in temperature.

With hotter filaments, more primary electrons become available for ionization of neutral gas molecules. This results in less power being needed to maintain ion saturation current (as measured by the Langmuir probes). The result of less required discharge power is a reduction in electron temperature, as shown in Fig. 3. The increase in electron density is attributed to the decrease in particle diffusion loss with lower electron temperatures.

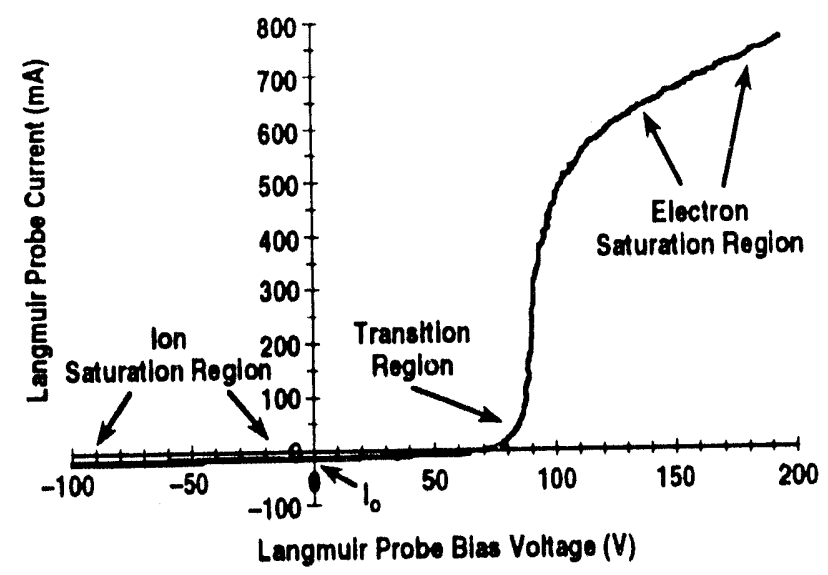

Fig. 2. Classical Langmuir probe current/voltage characteristic. 


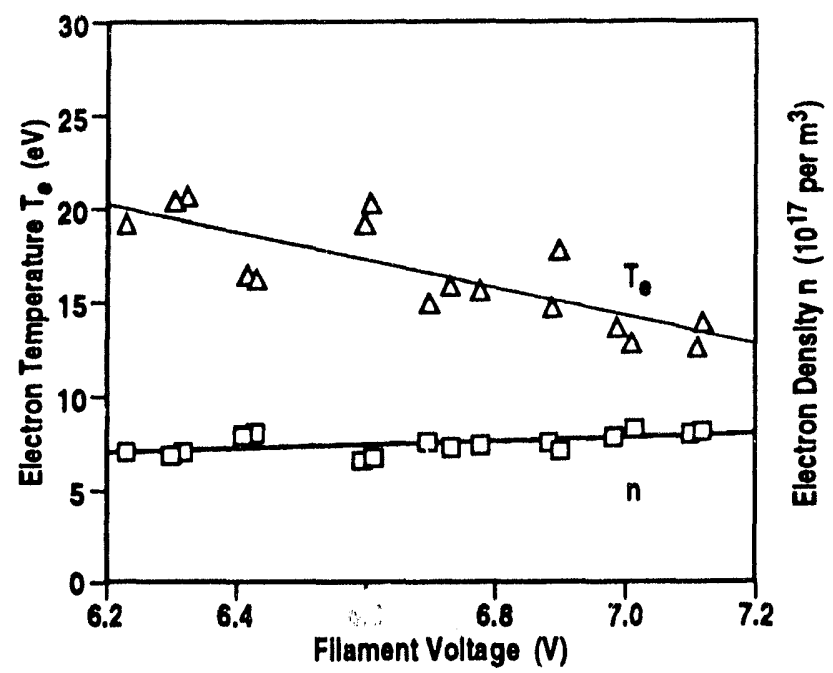

Fig. 3. Electron temperature and density for arc discharges at different filament voltages.

\section{EFFECT OF ARC POWER LEVEL}

As mentioned earlier, the arc power supply uses feedback regulation to maintain 8 . constant current feedback signal from a Langmuir probe biased with a fixed (negative) voltage. In effect, the power supply regulates the ion saturation current (measured by the Langmuir probe) within the ion source discharge. Since density is proportional to ion saturation current, the arc power supply can be considered a "plasma density controller" for the ion source, where the Langmuir probe signal command is "dialed in" to achieve the desired arc power level. Measured results (Fig. 4) indicate that arc power

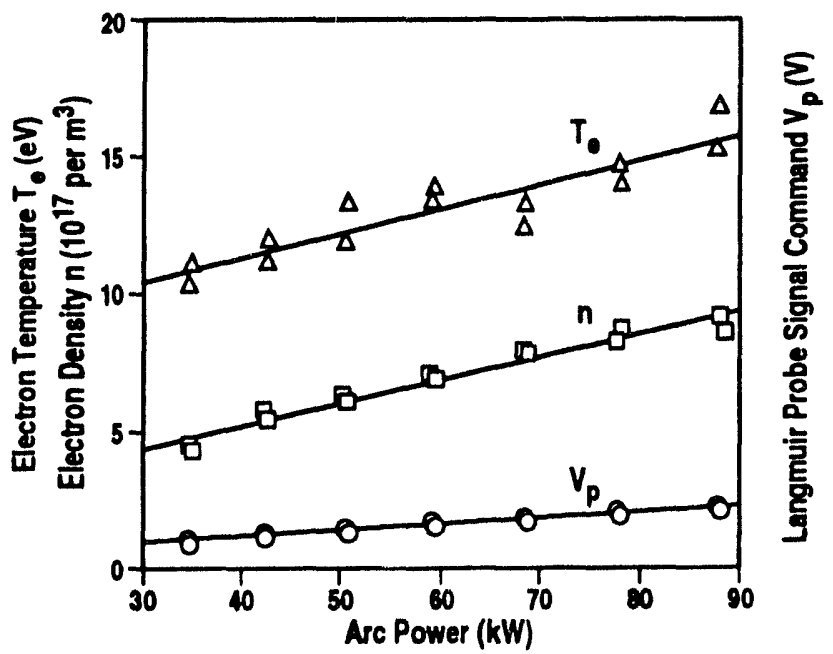

Fig. 4. Electron temperature, density, and Langmuir probe signal command for arc discharges at different arc power levels.
( $\left.I_{\text {arc }} V_{\text {arc }}\right)$ within the discharge is proportional to the Langmuir probe signal command.

To examine the effect of arc power level on electron temperature and density, a series of arc discharges was produced at various arc power levels, with filament voltage (and other ion source operation parameters) held constant. The results of temperature and density measurements are shown in Fig. 4, plotted against arc power. Both electron temperature and density are seen to increase linearly with arc power level. The high degree of linearity displayed by the plots of density and Langmuir probe signal command demonstrates that this arc power supply functions as a controller of plasma density in the ion source arc chamber. Furthermore, with a fixed filament voltage, the arc power supply must apply a higher voltage to the discharge to produce the higher requested density. The higher energies imparted to the primary electrons result in higher electron temperature after the discharge reaches thermal equilibrium.

\section{EFFECT OF BACKSTREAMING ENERGETIC ELECTRONS DURING A BEAM PULSE}

When beam is extracted from the ion source, the arc discharge may be affected by electrons streaming back into the arc chamber from the region between the accelerating grids. These electrons originate primarily from accelerated ions colliding with the grids, as well as collisions between accelerated ions and neutral gas molecules in this region. The electrons are accelerated back through the grids with energies proportional to the accelerating voltage. To determine the effect of these backstreaming energetic electrons on electron temperature and density within the arc chamber, beam extraction at various accelerating voltages is required. For comparison, a series of arc discharges (without beam extraction) was also produced, each discharge at the same ion source operation parameter settings as a corresponding beam extraction.

Electron temperature and density were measured under these conditions, and are shown in Figs. 5 and 6, respectively, plotted as functions of acceleration voltage. Both temperature and density are seen to increase with acceleration voltage level. However, the trends for data with and without beam appear to differ only slightly. This suggests that increases in temperature and density may be due solely to the increases in arc power corresponding to the increasing acceleration voltage (Langmuir probe signal command is increased for beams at higher energies, while filament voltage is maintained constant). The backstreaming energetic electrons may have little or no effect on the electron temperature and density inside the arc chaniber. (This can be explained by the relatively small collisional cross-section of energetic electrons with the plasma particles.) 


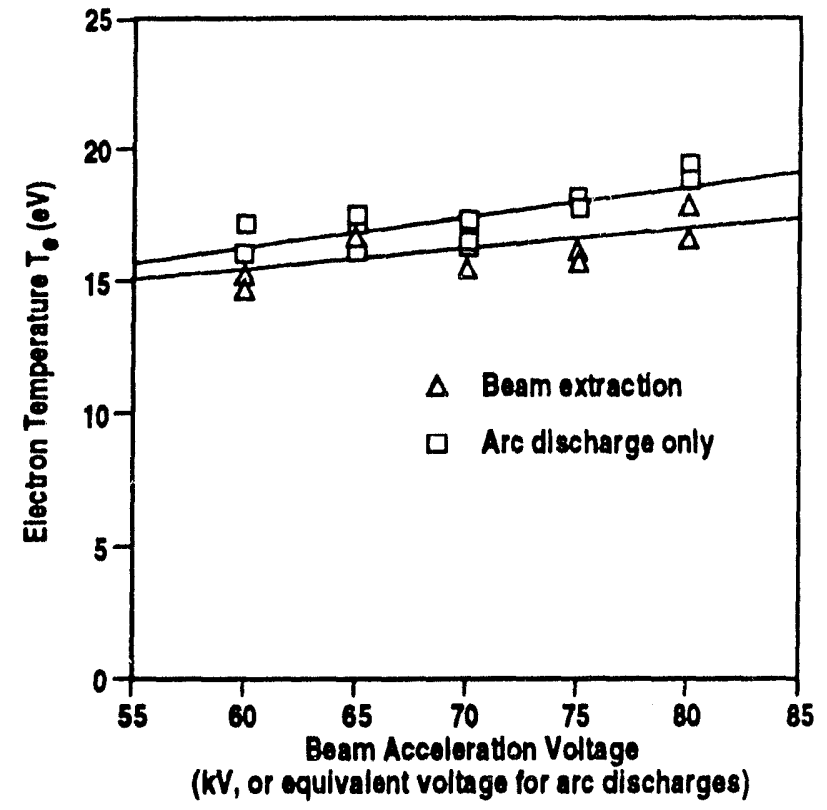

FIG. 5. Electron temperature for beam extractions at different acceleration voltages and for arc discharges at the same parameter settings as used during these beam extractions.

\section{TEMPERATURE AND DENSITY AS A FUNCTION OF TIME DURING A LONG BEAM PULSE}

To determine the extent that electron temperature and density vary over time during a beam pulse, Langmuir probe voltage sweeps were made every $0.5 \mathrm{sec}$ during a $3.5 \mathrm{sec}$ beam pulse. The resulting values of temperature and density indicate little change in these parameters throughout the pulse, the result of effective arc regulation using Langmuir probe feedback control.

\section{CONCLUSIONS}

Sweeping the Langmuir probe bias voltage to generate probe current/voltage characteristics provides new diagnostic capabilities for the DIII-D ion sources. The characteristics reveal that for arc discharges with fixed Langmuir probe signal commands, electron temperature decreases as filament voltage is increased, while electron density increases only slightly. For arc discharges with fixed filament voltage, both electron temperature and density increase linearly as arc power is increased. Extracting beam from the ion source appears to have little effect on electron temperature and density within the arc discharge compared against equivalent discharges with no beam extraction. Temperature and density increase with beam accelerating voltage, but this phenomenon

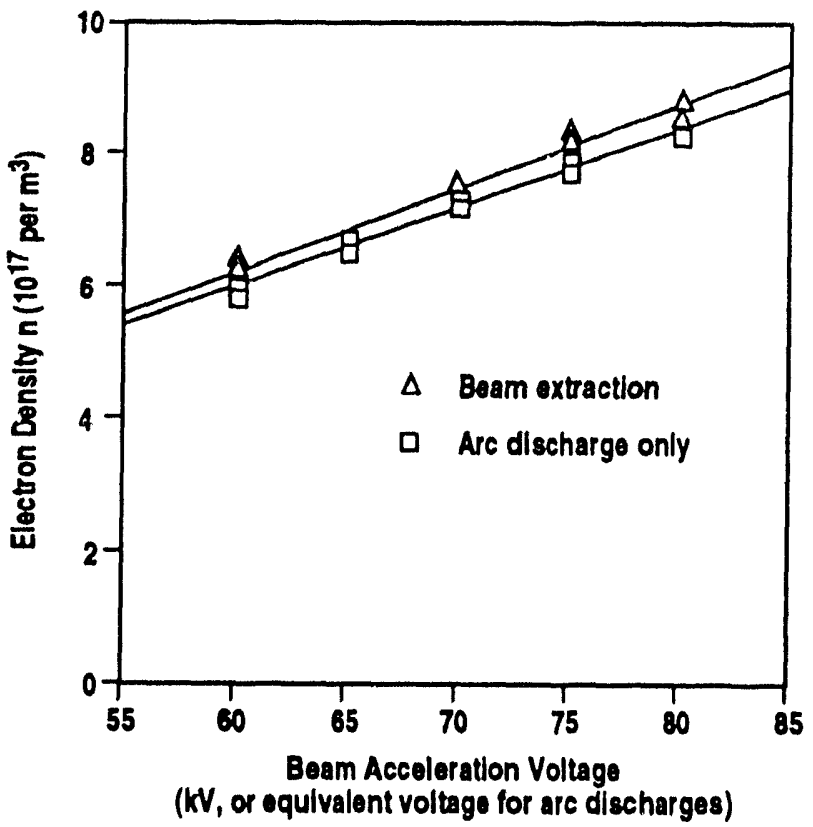

Fig. 6. Electron density for beam extractions at different acceleration voltages and for arc discharges at the same parameter settings as used during these beam extractions.

can be attributed to the increase in arc power necessary for operating the ion source with higher energy beams. Finally, temperature and density appear fairly constant over beam pulse durations of several seconds.

The results of these experiments help explain empirical observations and operational techniques developed for the neutral beam ion sources at DIII-D. This diagnostic will help characterize ion source operational regimes with respect to electron temperature and density. Troubleshooting operational problems may be aided by this tool as changes in source characterization are noted. Eventually, further improvements in operational technique will be developed as a result of information provided by this diagnostic.

\section{REFERENCES}

[1] R. Hong, A.P. Colleraine, J.S. Haskovec, D.H. Kellman, J. Kim, A. Neren, et al., "Operational experience with the DIII-D neutral beam $12 \mathrm{~cm} \times 48 \mathrm{~cm}$ common long pulse wource," in Proc. 12th IEEE Symp. on Fusion Engineering, pp. 1133-1136, 1887.

[2] R. Hong, D. Kellman, G. Santamaria, A. Colleraine, J. Kim, R. Lee, et al., "Beam current regulation of DIII-D neutral beam lang pulse ion sources," in Proc. 13th IEEE Symp. on Fusion Engineering, Pp. 887-800, 1988.

[3] 1.H. Hutchinnon, in Principles of Plasma Diegnostics. New York: Cambridge Univeraity Press, 1987, pp. 50-66. 

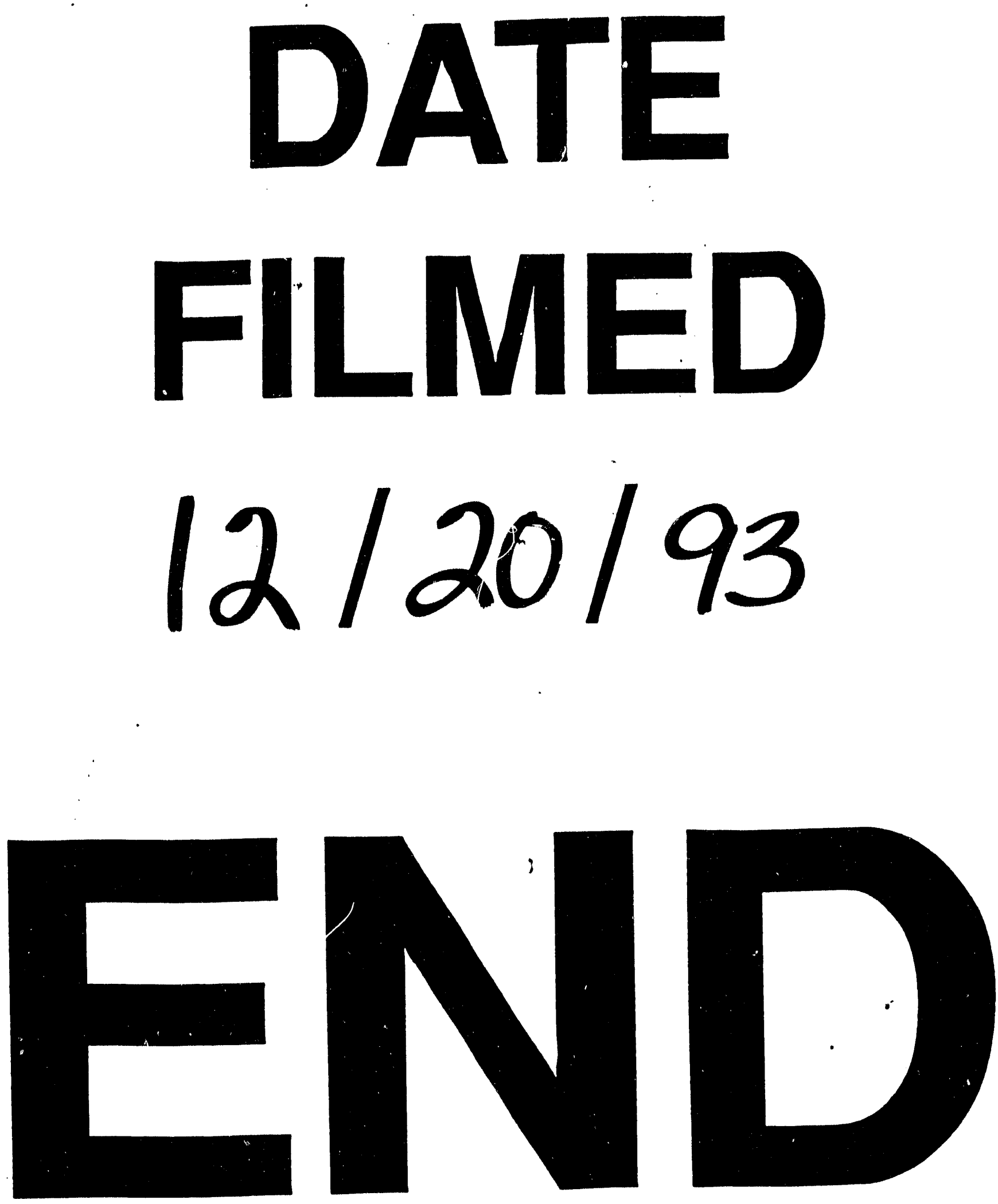
